\title{
OS EFEITOS DA EBSERH NA GESTÃO DOS RECURSOS HUMANOS DOS HOSPITAIS UNIVERSITÁRIOS FEDERAIS
}

\section{EBSERH'S effects on Human Resource Management of Federal University Hospitals}

Karina de Farias Pinheiro (UCSAL)

Ângela Maria Carvalho Borges (UCSAL)

Informações do artigo

Recebido em 15/06/2019

Aceito em 10/07/2019

doi>: https://doi.org/10.25247/2447-861X.2019.n247.p452-477

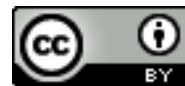

Esta obra está licenciada com uma Licença Creative Commons Atribuição 4.0 Internacional.

\section{Como ser citado (modelo ABNT)}

PINHEIRO, Karina de Farias; BORGES, Ângela Maria Carvalho. Os efeitos da EBSERH na Gestão dos Recursos Humanos dos Hospitais Universitários Federais. Cadernos do CEAS: Revista Crítica de Humanidades.

Salvador, n. 247, mai./ago., p. 452-477, 2019. DOI: https://doi.org/10.25247/2447-861X.2019.n247.p452-477

\begin{abstract}
Resumo
O artigo tem por objetivo analisar os efeitos da EBSERH na gestão dos recursos humanos dos Hospitais Universitários Federais que firmaram contrato de adesão com a Empresa Brasileira de Serviços Hospitalares - EBSERH. Para isso, o método de pesquisa escolhido foi a Revisão Sistemática de Literatura, que resultou na seleção e análise de 13 dissertações que sobre o tema, concluídas de 2014 a 2018 e disponibilizadas na Biblioteca Digital de Teses e Dissertações - BDTD/CAPES. Esses estudos demonstraram que os problemas de gestão dos recursos humanos nos Hospitais Universitários Federais, nos diversos Estados do país, são semelhantes, tanto no que diz respeito à recomposição parcial da força de trabalho quanto à presença de conflitos e diferenças legal-trabalhistas em face à diversidade de vínculos contratuais e às condições adversas de trabalho, resultando na fragmentação e fragilização dos trabalhadores. Concluiu-se que o novo modelo de gestão SUS EBSERH trouxe efeitos negativos para a gestão dos Recursos Humanos dos Hospitais Universitários Federais, uma vez que não cumpriu com as suas propostas iniciais, como a regularização da força de trabalho e promoção de melhorias na gestão.
\end{abstract}

Palavras-chave: EBSERH. Recursos Humanos. Gestão de Pessoal.

\begin{abstract}
The article aims to analyze the effects on the management of human resources in Federal University Hospitals that signed a contract with the Brazilian Company of Hospital Services EBSERH. For this, the research method chosen was the Systematic Literature Review, which resulted in the selection and analysis of 13 dissertations on the subject, completed from 2014 to 2018 and available at the Digital Library of Theses and Dissertations - BDTD / CAPES. These studies have shown that human resource management problems in Federal University Hospitals in the various states of the country are similar, both with regard to the partial rebuilding of the workforce and the presence of conflicts and legal-labor differences in relation to diversity of contractual links and adverse working conditions, resulting in the fragmentation and weakening of workers. It was concluded that the new SUS - EBSERH management model had negative effects on the Human Resources management of Federal University Hospitals, as it did not comply with its initial proposals, such as workforce regularization and promotion of management improvements.
\end{abstract}

Keywords: EBSERH. Human Resources. Personnel Management.

Os Hospitais Universitários Federais (HUF's) são instituições públicas vinculadas às Universidades constituindo o maior complexo hospitalar do País. Agregam duas importantes políticas públicas: a de Educação, responsável pela formação de profissionais de saúde e pela 
produção de pesquisas na área biomédica, e a política de Saúde, estando integrados à rede de serviços do Sistema Único de Saúde (SUS) como referência para média e alta complexidade (LITTIKE; SODRÉ, 2015).

Desde a década de 9o, os HUFs vinham sendo sucateados e precarizados pela falta de investimentos e restrições orçamentárias, redundando em uma crise manifesta nos déficits de recursos financeiros, tecnológicos e de recursos humanos, com repercussões negativas sobre o volume e a qualidade dos serviços prestados à comunidade. Essa crise, além de sucatear a infraestrutura, reduzir o número de leitos, levou ao elevado endividamento e à insuficiência de pessoal, provocada pela não contratação de novos servidores através de concursos públicos, como previsto no Regime Jurídico Único (RJU).

Esse estrangulamento na área de recursos humanos, segundo Druck (2016), está diretamente relacionado com a Lei de Responsabilidade Fiscal de 2000, que colocou a limitação das despesas com pessoal como um dos principais mecanismos de controle das contas públicas, focando nas despesas primárias - investimentos, políticas sociais, previdência, ciência e tecnologia dentre outros - para assegurar os recursos necessários ao pagamento do serviço da dívida, colocado como prioritário. A partir desta lei, os governos passam a restringir a contratação de pessoal pelo Regime Jurídico Único (RJU), através de concursos públicos e buscam suprir as necessidades de recursos humanos através de contratos atípicos, precários, temporários, contratação de empresas, cooperativas e ONGs para a execução direta de políticas sociais e, sobretudo no caso dos HUFs, para o fornecimento de mão de obra, introduzindo, por este mecanismo, trabalhadores terceirizados nas equipes responsáveis pela atividades-fim das instituições.

Essa repercussão nefasta da Lei de Responsabilidade Fiscal atingiu todos os níveis de governo e todos os setores. No caso dos HUFs, a terceirização de parte da força de trabalho necessária foi considerada irregular pelo TCU que, por meio do acórdão 1520/2006, recomendou a regularização da terceirização ilícita, já que os profissionais atuavam na prestação de serviços de saúde, portanto, nas atividades essenciais dos HUF's, as quais não podiam ser terceirizadas.

Essa recomendação não foi cumprida com a abertura de vagas através de concursos públicos, mas propiciou o retorno das propostas do Banco Mundial (BM), cujas diretrizes nortearam a Reforma do Estado nos anos go e a Lei da Responsabilidade Fiscal, em 2000. 
Em articulação com o Ministério de Educação (MEC), o Banco Mundial elaborou um diagnóstico situacional dos Hospitais Universitários Federaise constatou, segundo o relatório do Banco (GRAGNOLATI et al, 2013), que as instituições realizam procedimentos de altos custos, alta complexidade e tecnologia voltados para a medicina curativa; possuem uma má gestão, apresentam déficit nos recursos humanos somado a um contrato rígido de trabalho (Regime Jurídico Único); deterioração dos equipamentos e de todo campo tecnológico e da infraestrutura. Assim, fundamentado nesse diagnóstico, o BM apresentou, como melhor opção, para recuperar e sanar a crise dos HUF's a sua reestruturação (CISLAGHI, 2011), oU seja, o Programa Nacional de Reestruturação dos Hospitais Universitários Federais - REHUF, elaborado pelo BM com apoio do MEC e, posteriormente, a criação da Empresa Brasileira de Serviços Hospitalares (EBSERH).

O REHUF (Decreto no 7.082/2010), programa elaborado e financiado pelo BM, concedeu um empréstimo no valor de 756 milhões de reais, a serem divididos entre os HUF's existentes, os quais, em contrapartida, deveriam atender às condicionalidadesimpostas pelo BM, como, por exemplo, a adoção de um novo modelo de gestão (CISLAGHI, 2011, pg. 57).

A EBSERH foi criada na Lei Federal como Empresa Pública de Direito Privado, integrante da Administração Indireta, com patrimônio próprio, vinculada ao MEC, com prazo de duração indeterminado (art. $1^{\circ}$ da Lei no 12.550/2011). A sua finalidade é a prestação de serviços gratuitos de assistência médico-hospitalar, ambulatorial e de apoio diagnóstico e terapêutico à comunidade, assim como a prestação, às instituições públicas federais de ensino ou instituições congêneres, de serviços de apoio ao ensino, à pesquisa e à extensão, ao ensino-aprendizagem e à formação de pessoas no campo da saúde pública, de modo a preservar a autonomia universitária (art. $3^{\circ}$, caput, Lei nº 12.550/2011).

Por se constituir como uma Empresa Pública, a EBSERH deverá manter toda a prestação de serviços à saúde inserida integral e exclusivamente no âmbito do SUS, não excluindo a possibilidade de atenderindivíduos portadores de planos privados de assistência à saúde, desde que as despesas sejam ressarcidas a ela. Ad emais, a integralização do capital social será realizada com recursos oriundos de dotações consignadas no orçamento da União, bem como pela incorporação de qualquer espécie de bens e direitos suscetíveis de avaliação em dinheiro, pertencendo integralmente sob a propriedade da União ( $\left(1^{\circ}\right.$ e $3^{\circ}$ do art. $3^{\circ} ; \operatorname{art}^{\circ}$ da Lei de n 12.550/2011). Desta forma, a EBSERH deverá obedecer às orientações da Política 
Nacional de Saúde, às diretrizes e aos princípios do SUS, como a integralidade e universalidade na assistência, igualdade, equidade e a participação popular.

Assim, foi definido o financiamento compartilhado entre as áreas de educação e saúde (SODRÉ, 2013). A EBSERH, além de ser detentora de recursos do Estado, poderá ter outras fontes, como, por exemplo, receitas decorrentes de acordos e convênios com entidades nacionais e internacionais, criação de subsidiárias, aplicação financeira, receitas provenientes de serviços, alienação de bens e direitos, direitos patrimoniais sobre bens, doações que Ihe forem destinadas e rendas oriundas de outras fontes (Art. $8^{\circ}$ da Lei de $n^{\circ}$ 12.550/2011).

Quanto a forma de ingresso no quadro de pessoal da EBSERH, foi definid o o concurso público de provas ou de provas e títulos, sob o regime celetista, podendo ter contratos por tempo indeterminado e temporário, desde que a soma dos 2 (dois) períodos não ultrapasse 5 (cinco) anos (Arts.10 e 11 da Lei $n^{\circ}$ 12.550/20111). Em regra, os concursos são destinados a unidades hospitalares específicas, mas isso não impede que, diante da necessidade ou interesse da Empresa, possa haver a transferência para outra filial da EBSERH. Ainda, os empregados públicos da EBSERH terão planos de Cargos, Carreiras e Salários e de Benefícios da empresa (SITE EBSERH, Cartilha perguntas e respostas 2018). A lei que criou a EBSERH previu ainda que o quadro de pessoal da empresa também pode ser composto pelos servidores públicos estatutários - a maior parte dos que já compunham as equipes do HUFs - os quais são cedidos para a realização de atividades de assistência à s aúde e administrativas (Art. $7^{\circ}$ da Lei de no 12.550/2011).

A adesão à EBSERH não ocorreu de modo automático, ou seja, a partir da promulgação da referida Lei. Foi necessário que houvesse interesse por parte da Universidade Federal à qual o hospital é vinculado. Somente a partir da manifestação de interesse é celebrado um contrato de adesão entre as partes, no qual são estabelecidas as obrigações dos signatários, as metas de desempenho, os indicadores e prazos de execução que deverão ser observados pelos contratantes e a respectiva sistemática de acompanhamento e avaliação, com os critérios e parâmetros a serem aplicados (Art. $6^{\circ} \mathrm{da}$ Lei de no 12.550/2011). Após a celebração do contrato, a EBSERH, em conjunto com o hospital, realizarão o diagnóstico situacional da instituição com vistas a traçar um planejamento individualizado que melhor atenda à superação das condições apontadas 
como deficientes. Essas informações farão parte do Plano de Reestruturação, que será um dos anexos do contrato (SITE EBSERH, Cartilh a de perguntas e respostas, 2018).

Segundo dados da página oficial daEBSERH (2019), dos 50 HUF's vinculados a 35 IFES, 40 deles já celebraram com a EBSERH o contrato de adesão, restando apenas g HUF's sem contrato. Dos nove hospitais sem contrato, oito pertencem à Universidade Federal do Rio de Janeiro (UFRJ), que decidiu não fazer parte da rede e um pertence à Universidade Federal do Rio Grande do Sul (HCPA/UFRGS), cujo Hospital Universitário - Hospital de Clínicas de Porto Alegre - já possui natureza jurídica de empresa pública, portanto, está impedido de ser administrado por outra empresa. Já o Hospital Universitário da Universidade Federal de São Paulo (HU/UNIFESP) realiza atendimentos de caráter particular, por isso, não pode integrar a rede EBSERH.

Desde a sua proposta, essa opção de reestruturação dos HUFs foi amplamente criticada pelos funcionários e pelos pesquisadores que tratam do tema. O diagnóstico elaborado pelo BM foi considerado simplista e genérico, atendo-se apenas aos aspectos custos-benefícios (CORREIA, 2007). Ou seja, ele afasta a relevância social e política da maior rede hospitalar de serviços de saúde pública, fortalecendo o discurso de que o setorprivado seria mais eficiente na prestação de serviços de saúde do que o setorpúblico e entende que o Estado deve atuar apenas como regulador e financiador do sistema.

Ademais, observou-se que o Programa do BM/MEC relativiza a autonomia das Universidades, trazendo consigo a produtividade medida por quantidade e a flexibilização de contratos e direitos trabalhistas, o fim de licitações e prestações de contas, a flexibilização de currículos na formação dos profissionais de acordo com os interesses do mercado e fim da pesquisa pública (CISLAGHI, 2010, p. 7).

Também Druck (2016), observa que a EBSERH é uma empresa pública com fins lucrativos que irá apropriar-se de todo patrimônio físico e do conhecimento de um Hospital Universitário, alijando as Universidades Federais de sua gestão. Cislaghi (2011) compartilha do mesmo entendimento, considerando que a criação da EBSERH foi uma estratégia utilizada para transferir a gestão dos HUF's das Universidades para a empresa pública, o que implicou a centralização dos serviços de saúde e o deslocamento da saúde da ordem social na Constituição, passando a ser regulada pelos critérios da ordem econômica, sob os ditames do mercado. 
Também a Associação Nacional dos Dirigentes das Instituições de Ensino Superior ANDIFES, embora reconhecendo a necessidade de implementar um novo modelo de gestão nos UHFs, diante do quadro de precarização do trabalho, subfinanciamento e a autonomia restrita dos Hospitais Universitários, colocou em questão qual seria o modelo ideal, pois, no momento da aplicação do REHUF, havia uma rejeição da comunidade acadêmica e dos Conselhos de Saúde ao modelo de gestão inicialmente proposto para os Hospitais Universitários - as Fundações Estatais de Direito Privado - FEDP -, (CISLAGHI, 2011). Tal rejeição foi explicitada na $14^{\mathrm{a}}$ Conferência Nacional de Saúde (CNS) quando foi emitida uma moção de repúdio contra toda e qualquer forma de privatização do SUS, colocando em relevo as novas modalidades de gestão, tais como: Organizações Sociais (OS), Organizações da Sociedade Civil de Interesse Público (OSCIP), Fundações Estatais de Direito Privado (FEDP), Empresa Brasileira de Serviços Hospitalares (EBSERH), por implicarem a desconsideração dos princípios e diretrizes do SUS, a precarização do trabalho, o desvio os recursos públicos para o setor privado e a distorção das regras da administração pública em favor do capital (14 $\left.{ }^{\mathrm{a} C N S}, 2012\right)$.

Os críticos observaram, ainda, que a gestão e administração dos recursos financeiros do Programa REHUF, destinados à reestruturação e revitalização dos Hospitais das Universidades Federais integrados ao Sistema Único de Saúde(SUS), foram colocados sob a responsabilidade da EBSERH, com o que, mesmo os HUF's que não tenham celebrado com ela o contrato de adesão, ficaram subordinados à empresa em razão da dependência de recursos financeiros que são imprescindíveis para a manutenção das instituições (CISLAGHI, 2011; ZIGATO, 2015). Diante deste cenário, Cislaghi, (2010) afirma que a reforma da gestão dos HUF's implica a continuidade da lógica da Reforma do Estado, onde os serviços de saúde se enquadram no setor das atividades não exclusivas do Estado, de modo que seja possível realizar a gestão de um serviço público por um ente privado. $E$, ainda, ressalta que o $B M$ não quer apenas reformar a gestão, mas, sobretudo, tem a intenção de extinguir os HUF's ou substituí-los por empresas privadas, desvinculando-os das IFES, bem como do SUS.

No debate sobre a privatização dos HUFs com a criação da EBSERH, considere-se incialmente a discussão de Di Pietro (2017), que ressalta que a privatização é um conceito em aberto, podendo ser interpretada de forma restrita ou ampla. Mas, ambas as formas têm o mesmo objetivo que é reduzir o tamanho do Estado, fortalecer a iniciativa privada e os modos de gestão dos serviços públicos. Assim, a privatização restrita ou clássica se traduz na 
alienação das empresas públicas ao setor privado, com transferência de titularidade. Há uma mercadorização dos serviços públicos, em que os usuários se tornam clientes ou consumidores, detentores da liberdade de escolha de fornecedor, passando o serviço público a ser uma figura subsidiária do mercado. Enquanto a privatização ampla revela-se por meio da desregulação, desmonopolização de atividades econômicas, concessão, parcerias públicas e privadas e pelos contratos de gestão.

Para Sodré (2013), a EBSERH é uma forma velada de privatização, porém não se enquadra na privatização clássica ou privatização no sentido técnico, porque não implica delegação de serviços, tampouco a desconstituição da natureza de serviço público dos serviços do SUS prestados em suas dependências. Também Granemann (2011) considera que a privatização das políticas sociais do Brasil, como a saúde, caracteriza-se como uma privatização não-clássica, revelando-se pela transferência do fundo público para diferentes modelos de instituições privadas. Correia (2011) considera que, no setor da saúde pública, a privatização se efetiva através do repasse da gerência e da gestão de serviços e de pessoal deste setor para grupos privados por meio dos "contratos de gestão" e de "termos de parcerias", mediante transferência de recursos públicos. A entrega dos Hospitais Universitários à EBSERH permite que a lógica do setor privado seja predominante neste espaço, de modo a priorizar os interesses do mercado privado da educação e da saúde, da indústria de equipamentos e medicamentos (CORREIA, 2011). Finalmente, SANT' HELENA et al (2013) destacam a perda de autonomia universitária, a submissão da produção de conhecimento e formação de profissionais aos interesses mercantis e, por consequência, a não ampliação ao acesso dos usuários à rede de saúde e mitigação dos seus direitos, transformando a saúde em um espaço de lucratividade para o mercado.

Finalmente, quanto aos recursos humanos e particularmente às formas de contratação, considera-se que este é um dos pontos chave para a avaliação dos resultados alcançados pela EBSERH. Isto porque, como referido, a criação dessa empresa foi pautada na justificativa da necessidade de reestruturar e modernizar os Hospitais Universitánios Federais, sobretudo recompora força de trabalho que, segundo os relatórios apresentados pelo Tribunal de Contas da União (TCU) estava irregular diante das terceirizações ilícitas e precárias, contrariando a súmula 331 do Tribunal Superior do Trabalho (TST), que não autorizava a terceirização das atividades fim na prestação dos serviços de saúde, 
irregularidade que se evidenciava também no não cumprimento do princípio constitucional da seleção mediante concurso público.

Quanto a esse ponto, os críticos ressaltam que, com o EBSERH observa-se a continuidade da flexibilização nos contratos de trabalho com a aplicação das regras do direito privado (SANT' HELENA et al, 2013; DI PIETRO, 2017) em detrimento do Regime Jurídico Único que rege os contratos do funcionalismo público.

Essa é a temática que será discutida a partir daqui, com base nos resultados de um estudo ${ }^{1}$ que teve por objetivo analisar, através da metodologia da Revisão Sistemática de Literatura (RSL), os efeitos na gestão dos recursos humanos dos Hospitais Universitánios Federais que aderiram à Empresa Brasileira de Serviços Hospitalares, identificando se a multiplicidade de vínculos contratuais, RJU e CLT, diagnosticada antes da implantação da EBSERH, interfere na gestão de pessoal, bem como se a EBSERH superou o déficit da força de trabalho, por meio de novas contratações e, ainda, em que medida ocorreu a substituição dos funcionários contratados via Fundações de Apoio.

\section{Metodologia}

A escolha da RSL como método para atingir os objetivos propostos partiu da constatação, no processo de levantamento bibliográfico, da existência de uma produção científica considerável sobre a temática, com destaque para os estudos dos seguintes autores: LITTIKE (2013, 2015), SODRÉ (2013, 2015), CISLAGHI (2010, 2011), ANDREAZZI (2013), CORREIA (2007, 2011), DRUCK (2016)，GRANEMANN (2011), os quais abordaram vários aspectos sobre a criação da EBSERH, seu papel na gestão dos Hospitais Universitários Federais e as controvérsias sobre tal política.

Além disso, como afirma Faria (2016), a RSL consiste em um método transparente, sistemático e explícito, com rigor científico e metodológico na identificação, seleção e avaliação crítica dos dados bibliográficos. É um tipo de estudo que possibilita concentrar diversos trabalhos referentes a um mesmo assunto, em um único documento, de modo a obter um estado da arte atualizado, além de constituir uma contribuição mais relevante do

\footnotetext{
${ }_{1}^{1}$ PINHEIRO, K.F. Os Efeitos da EBSERH na Gestão dos Recursos Humanos dos Hospitais Universitários Federais. Dissertação (Mestrado em Políticas Sociais e Cidadania) - Universidade Católica de Salvador (UCSAL), Salvador, 2019 .
} 
que a realização de mais um estudo de caso de uma EBSERH em particular, baseado em algumas entrevistas e necessariamente limitado, face às dificuldades encontradas para o acesso de dados nesta área.

Seguindo o protocolo da RSL (FARIA, 2016), após a formulação do problema de pesquisa e a definição dos objetivos, foi definida a base de dados a ser pesquisada, recaindo a escolha sobre a Biblioteca Digital de Teses e Dissertações - BDTD / CAPES (http://bdtd.ibict.br/), que agrega teses e dissertações produzidas nos programas de pósgraduação no Brasil, reconhecidos pela CAPES. A escolha de dissertações - não foram encontradas teses sobre o tema - justifica-se por serem estudos de qualidade controlada e com reconhecimento científico e acadêmico, sendo, portanto, fontes primárias seguras e qualificadas.

Quanto ao período selecionado para o estudo, estabeleceu-se a delimitação de 2014 como ano inicial, considerando-se a data de criação da EBSERH, final de dezembro de 2011, o lapso temporal para a celebração do contrato de adesão entre a Empresa e as Instituições de Ensino Superior e a implementação do planejamento estratégico nos Hospitais Universitários Federais. A definição de 2018 como ano final buscou capturar o maior número possível de trabalhos. A escolha do idioma português, decorreu, naturalmente, da base de dados selecionada, que contém apenas estudos realizados no Brasil, no contexto da pós graduação nacional.

Definidos esses parâmetros iniciais, foram estabelecidos os critérios de inclusão e exclusão, a estratégia de busca das pesquisas, a metodologia de coleta e a síntese dos dados.

Quanto à definição dos critérios de inclusão e exclusão, foram incluídas dissertações publicadas no período de 2014 a 2018, que trataram sobre a gestão dos recursos humanos, como as relações contratuais e condições de trabalho nas Empresas Brasileiras de Serviços Hospitalares - EBSERH e excluídas as dissertações que, mesmo focando na EBSERH, não abordaram as questões de recursos humanos e/ou que não estiveram dentro do período especificado - 2014 a 2018.

A estratégia de busca incluiu a identificação das teses e dissertações que atenderam aos critérios de inclusão e a posterior seleção daquelas que iriam constituir a base para o estudo.

Na etapa de busca, a pesquisa realizada na Biblioteca Digital de Teses e Dissertações - BDTD/CAPES seguiu os seguintes parâmetros e as seguintes palavras -chave:(a) EBSERH/ 
assunto; (b) EBSERH/Título; (c) EBSERH e Força de trabalho/ todos os campos/ busca avançada; (d) EBSERHe gestão de Recursos Humanos / todos os campos / busca avançada e (e) EBSERH e relações de trabalho/todos os campos/busca avançada, com o intuito de conseguiro maior número de resultados possíveis.

Utilizando esses termos de pesquisa supracitados, na base de dados selecionada, foram identificadas ${ }^{2} 52$ dissertações - (EBSERH/assunto: 13 dissertações; EBSERH/título: 16 dissertações, EBSERH e Força de trabalho/todos os campos/busca avançada: 6 dissertações; EBSERH e gestão de Recursos Humanos / todos os campos / busca avançada: 10 dissertações e, EBSERH e relações de trabalho/todos os campos/busca avançada: 7 dissertações - e observou-se que, deste total, 27 dissertações estavam replicadas, ou seja, uma mesma dissertação apareceu na pesquisa com diferentes palavras-chave.

Portanto, quando excluídas do total, restaram apenas 25, as quais passaram pelas etapas de exclusão e inclusão, e posteriorseleção, coleta e análise.

Os 25 trabalhos identificados nesta etapa foram todos registados em uma tabela com as seguintes informações: título, autor/data, área de conhecimento, Programa de PósGraduação (PPG) e a Instituição de Ensino Superior (IES), fonte (endereço na Web), metodologia, objetivo e conclusão e justificativa da exclusão.

Os estudos excluídos foram aqueles que não atenderam aos critérios de inclusão, uma vez que não tinham relação com o objetivo da pesquisa ou não se enquadravam ao período proposto, publicações de 2014 a 2018. Enquanto, os estudos incluídos foram aqueles que, numa primeira aproximação, atenderam aos critérios preestabelecidos, mas que ainda passariam por uma análise mais criteriosa, podendo ser selecionados ou não.

Portanto, a pesquisa realizada com base nesta metodologia permitiu uma captura mais ampla de dados, tendo exigido, um refinamento posterior, para conferir se todos os trabalhos identificados estavam efetivamente relacionados com os objetivos da pesquisa. $O$ primeiro refinamento consistiu na leitura dos Resumos, o que possibilitou uma tomada de decisão mais segura para os incluir ou não na bibliog rafia final.

2 ESTUDOS IDENTIFICADOS: estudos identificados são todos aqueles encontrados na base de dados da Biblioteca Digital de Teses e Dissertações - BDTD a partir da pesquisa com as palavras-chave. 
Após esta etapa, do total de 25 dissertações resultantes da etapa de busca, foram excluídas 7 dissertações e 18 permaneceram incluídas. Contudo, esse refinamento ainda não foi suficiente para apurar os trabalhos que, de fato, interessavam ao p resente estudo.

Em um novo refinamento, com a leitura completa dos estudos, das 18 dissertações Incluídas inicialmente, 5 não foram selecionadas devido aos seguintes motivos: na dissertação (D14)3 , embora o estudo tenha abordado a gestão de pessoal após a implantação daEBSERH, os dados foram insuficientes. Isto, porque a data da celebração do contrato entre a EBSERH e a UFSC ocorreu em 16 de março de 2016 e a pesquisa, ora analisada, também foi publicada próximo a este período, agosto de 2016. Portanto, não houve tempo hábil para uma análise mais precisa, razão pela qual a autora se ateve mais às questões anteriores à EBSERH; o estudo (D15) 4demonstrou que a presença da EBSERH nos HUF's enfraqueceu o controle social, impossibilitando a efetiva participação de controle e implementação das políticas públicas de saúde; na dissertação (D16) 5, o autor investigou o desempenho hospitalar sob dois aspectos: assistencial e de ensino; o estudo $\left(D_{17}\right)^{6}$ apontou que a EBSERH se afastou dos princípios do SUS, uma vez que se aproximou da lógica do Estado Capitalista Ultraliberal, não abordand o questões relacionadas à gestão dos recurso s humanos e o estudo (D18) 7 não estava disponível na integra para devida análise, embora tenha focado na satisfação e insatisfação no trabalho dos profissionais do Hospital Universitário de Brasília.

Deste modo, na segunda etapa do refinamento - leitura do resumo, introdução, metodologia e conclusão -, de alguma forma, os estudos dialogaram com os objetivos e o problema de pesquisa; das 18 dissertações incluídas inicialmente, 13 dissertações foram finalmente selecionadas para constituírem a base das últimas etapas do estudo (sistematização e análise dos dados).

3 PAULA, R.B. DE. Desafios de Gestão do HU/UFSC, após a criação da EBSERH pelo Governo Federal, Universidade Federal de Santa Catarina, 2016.

4 ALBUQUERQUE, G. O. Debate sobre a EBSERH em instâncias de controle social: um estudo exploratório. Universidade de Brasília (UnB), 2017.

5 ORLANDI, Daniele de Paula. Análise dos impactos da EBSERH no desempenho dos Hospitais Universitários Federais. Universidade Federal do Espírito Santo, 2016.

6 OLIVEIRA, G. DE A. A compatibilidade dos princípios e modelo de estado que subjazem ao SUS e a EBSERH. Dissertação Faculdade de Filosofia da Pontifícia Universidade Católica do Rio Grande do Sul, 2014.

7 BRITO, L.M. DE. Satisfação e insatisfação no trabalho de profissionais em Hospital Universitário. Universidade de Brasília, 
Essas 13 dissertações $\left(D_{1} \text { a } D_{13}\right)^{8}$ foram lidas integralmente para a coleta das informações de interesse da pesquisa, as quais foram sintetizadas em uma segunda tabela, constituída pelos seguintes elementos: Título; autor/data; fonte (endereço eletrônico da Web); metodologia; objetivo e conclusão; e o enfoque dos estudos direcionado aos recursos humanos sob a gestão da EBSERH. A construção dessa tabela possibilitou a organização dos dados coletados de maneira clara e sucinta, permitindo uma melhor visualização do que se buscava, além de ter facilitado a análise e interpretação das dissertações selecionadas.

\section{Análise dos dados}

As 13 dissertações selecionadas trataram sobre os impactos trazidos na gestão dos Recursos Humanos dos HUF's que aderiram à EBSERH, pesquisaram os HUF's de diversos Estados do país - Espírito Santo - UFES (D1e D12); Minas Gerais - UFJF (D2, D9), UFTM (D3), UFU (D10); Rio de Janeiro - UFF (D4); Brasília /DF - UNB (D8); Rio Grande do Norte - UFRN (D5, D6, D11); Pernambuco - UFPE (D7); Santa Catarina - UFSC e Rio Grande do Sul - UFSM

8 AVELAR, J.V.R. Disputa de Frames e Hibridismo Organizacional um conteúdo de caso de um Hospital Universitário Federal. Universidade Federal do Espírito Santo - UFES. Vitória/E. S, 2016 (D1)

TEIXEIRA, Enio Henrique. Um sistema de qualidade para a gestão compartilhada do Hospital Universitário da UFJF com a EBSERH. Universidade Federal de Juiz de Fora, 2016.. (D2)

MAZETO, Y.E.S. Política de saúde face aos avanços neoliberais e a implantação da Empresa Brasileira de Serviços Hospitalares. PUC-GOIAS, 2017.. (D3)

SILVA, J.A. Empresa Brasileira de Serviços Hospitalares: coerência ou contradição de um Estado empresário? Universidade Federal Fluminense, 2016. (D4)

VIEIRA, K.R. Transformações estruturais e institucionais da gestão do HULW/UFPB. Universidade Federal do Rio Grande do Norte, 2017. (D5)

GURGEL, J.L.M. Análise de desempenho: um estudo aplicado em um Hospital Universitário do Rio Grande do Norte conveniado à EBSERH. Universidade Federal do Rio Grande do Norte, 2016. (D6)

BARROS, R.T. Empresa Brasileira de Serviços Hospitalares: uma análise sobre sua gestão no contexto institucional do Hospital das Clínicas da UFPE. Universidade Federal de Pernambuco, 2014. (D7)

FERNANDES, R.B. Da Conquista ao Desmonte: um estudo sobre os descaminhos da política de saúde em tempos de EBSERH. Universidade de Brasília, 2017. (D8)

ZIGATO, J.G. A Implantação da Empresa Brasileira de Serviços Hospitalares (EBSERH) no Hospital Universitário. Universidade Federal de Juiz de Fora2015. (Dg)

FLAUSINO, V.S. Cultura e poder na organização hospitalar: as relações de poder na implantação da EBSERH e um Hospital Universitário. Universidade Federal de Uberlândia - UFTM, 2015. (D10)

PESSOA, C.M. Cultura Organizacional e Condições de Trabalho: impactos da contratualização de um Hospital Universitário com a EBSERH Universidade Federal do Rio Grande do Norte,2018. (D11)

MARINHO, R.N. Implicações estruturais e comportamentais na implementação do modelo de gestão da EBSERH em um Hospital Universitário. Universidade Federal do Espírito Santo, 2016. (D12)

CARMINATI JR, A. A gestão do Hospital Universitário da Universidade Federal de Santa Maria e da Universidade Federal de Santa Catarina após a adesão à EBSERH. Universidade Federal de Santa Catarina, 2017 (D13). 
(D13). Ressalta-se que dez delas utilizaram a metodologia do estudo de caso e três (D2), (D4) e (D6) optaram pela análise documental.

Então, pode-se verificar que a diversidade regional dos estudos trouxe uma visão ampliada sobre a gestão da EBSERH nos HUF's, principalmente, no tocante à gestão de pessoal. As informações nelas contidas demonstraram que os problemas são semelhantes em relação à recomposição da força de trabalho, relação contratual e condições de trabalho.

Neste sentido, para a análise dos dados foram determinadas três categorias. Na categoria Força de Trabalho examinou-se se, de fato, houve a recomposição de pessoal por meio de novas contratações e cessão dos servidores estatutários à EBSERH e a substituição dos trabalhadores terceirizados via Fundações de Apoio. Já na categoria Relação Contratual buscou-se identificar os vínculos de trabalho existentes nos HUF's a partir da adesão à EBSERH, bem como menções sobre as diferenças legal-trabalhistas e as questões relativas às relações de poder. Finalmente, na categoria Condições de Trabalho buscou-se examinara presença de conflitos no ambiente laboral, o adoecimento dos funcionários/servidores, a fragmentação e fragilização da classe trabalhadora, a comunicação entre os pares e as transformações decorrentes da introdução da gestão gerencial. Como será mostrado, esta categoria é o reflexo das demais, pois as mudanças de gestão e contratuais influenciaram no processo de trabalho e na vida dos trabalhadores.

\section{Força de trabalho}

De acordo com os estudos selecionados, antes da EBSERH, era notória a deficiência de pessoal nos HUF's, decorrente das aposentadorias, falecimentos, afastamentos para tratamentos de saúde e da não reposição efetiva destes postos de trabalho, gerando fechamento de leitos, redução e queda na qualidade dos atendimentos, bem como a presença dos funcionários terceirizados irregulares.

Após a celebração do contrato entre a EBSERH e as IFES, os estudos demonstraram que os HUF's realizaram novas contratações via concurso público de provas, ou de provas e títulos e, implicitamente, no discurso dos autores, houve também a cessão dos servidores públicos estatuários à Empresa, quando mencionaram a presença da duplicidade de vínculo contratual nos HUF's - CLT/EBSERH e RJU, de modo que aumentou o quantitativo de trabalhadores nestas instituições. 
Contudo, em muitos HUF's do país, a exemplo HU-UFJF (D2), HU-UFTM(D3) e (D10), os autores, de modo explícito, indicaram que a reestruturação da força de trabalh o ocorreu parcialmente, ainda permanecendo um déficit quantitativo no quadro de pessoal. Inclusive, no HU-UFJF (D2), não havia o diagnóstico situacional sobre a quantidade de pessoal por setor, anterior à realização do certame público EBSERH. Isto é aponta do como uma falha grave, pois é prevista no contrato EBSERH e IFES, como requisito obrigatório, a produção do diagnóstico situacional do HUF, a fim de se traçar um planejamento individualizado que melhor atenda às condições apontadas como deficientes, de maneira que essas informações se tornem parte do Plano de Reestruturação da instituição (SITE EBSERH, Cartilha de Perguntas e Respostas, 2018).

No HU-UFTM D10, o autor questionou a efetividade da EBSERH, já que foi criada sob a justificativa de regularizar e recompor a força de trabalho, mas não o fez plenamente.

Já nos Hospitais Universitários - HULW - UFPB (D5); HUCAM - UFES (D12) e o HUUFSM (D13), a EBSERH substituiu totalmente os funcionários terceirizados via Fundação de Apoio. Contudo, a convivência dos regimes celetistas e estatutários é tida como um dos pontos mais conflitantes e limitador para a gestão EBSERH, principalmente, segundo o estudo (D10), em razão de os funcionários da EBSERH serem vistos como o "futuro" do hospital, o que os coloca em uma posição superiorà ocupada pelos demais profissionais não EBSERH, evidenciando problemas decorrentes da coexistência de trabalhadores contratados sobre regimes diferentes e, até mesmo, a fragmentação do departamento pessoal em dois, um para cada vínculo (D1).

No HU-UFSC (D13), o autor ressaltou que a EBSERH ainda não havia cumprido nenhuma das obrigações contratuais até a data do término do seu estudo. Os leitos continuavam fechados e nenhum funcionário aprovado no concurso público realizado em 2016 tinha sido convocado.

Como dito anteriormente, a terceirização da Força de Trabalho dos HUF's foi o que ensejou, a princípio, a criação da EBSERH, surgindo com o propósito de recompor o quadro de pessoal, ora defasado, bem como regularizar as contrataçõesilícitas. Mas tal regularização ainda não ocorreu de forma plena na maioria dos HUF's, portanto, eles não estão cumprindo a sua missão inicial, mesmo após de tantos anos da aprovação da Lei de nº 12.550/2011 e dos contratos celebrados com as IFES. 
Segundo relatório do TCU (TCo32-519/2014-1), a EBSERH está realizando a substituição dos terceirizados de forma gradual, em razão da não captação de profissionais em algumas especialidades, principalmente, na área médica. Desta forma, a permanência dos empregados terceirizados é justificada pela necessidade de evitar a interrupção dos serviços; o fechamento de leitos com uma possível demissão em massa dos terceirizados; para permitir a transferência de conhecimentos para os novos concursados, pois não possuem a devida experiência prática requerida pela instituição - HUF, bem como a fim de evitar o impacto financeiro decorrente das futuras demissões dos trabalhadores irregulares.

Assim, o estudo (D10) demonstrou, através de entrevistas sobre a realidade do HCUFTM, que a permanência dos terceirizados - FUNEPU se deve a duas causas centrais: a falta de recursos para rescindir os contratos de trabalho, de modo que os funcionários passarama ser pressionados a pedir demissão a fim de minimizar os valores pagos com a rescis ão, e a necessidade da transferência de conhecimento técnico e institucional para os funcionários EBSERH. Na fala dos trabalhadores terceirizados, toda esta situação é muito humilhante, pois sabem que, após os treinamentos, serão descartados. E, de forma contraditória, os terceirizados são vistos como inaptos e incompetentes para a realização do trabalho, uma vez que não conseguiram ingressar no $\mathrm{HU}$ através do concurso público realizado pelo EBSERH, tornando-se um grupo de trabalhadores minoritário, com curto tempo de vida no interior desta instituição e, por isso, muitas vezes, são preteridos pelos demais profissionais.

\section{Relação contratual}

$\mathrm{Na}$ categoria Relação Contratual foram identificados os vínculos de trabalho existentes nos HUF's sob a gestão da EBSERH, as diferenças legal-trabalhistas e quem exerce o poder diretivo-comando nestas instituições.

De acordo com os estudos selecionados, no período estudado, na maioria dos HUF's estavam presentes três vínculos distintos, a saber: os servidores estatutários re gidos pelo RJU; os empregados EBSERH regidos pela CLT e os terceirizados, também, regidos pelo regime privado. Apenas nos Hospitais Universitários - HULW/UFPB (D5), HUCAM/UFES (D1 E 12) e HUFSM (D13) -, onde houve a completa substituição dos funcionários terceirizados, restaram apenas os outros dois vínculos.

Os estudos, de forma unânime, apontaram que o contexto híbrido da Força de Trabalho nos HUF's ocasiona conflitos e fragmentação da classe trabalhadora, dividindo-a 
em dois blocos principais: o bloco dos RJU e o bloco dos celetistas EBSERH, devido às diferenças legal-trabalhistase às mudanças na gestão.

Assim, o estudo D1 apontou as principais diferenças legal-trabalhistas entre os servidores RJU e os celetistas EBSERH observadas no HUCAM/UFES. Vejamos: (a) vínculos empregatícios distintos para a mesma função e espaço laboral (RJU e CLT); (b) carga horária diferente para cada vínculo (RJU - 30 horas semanais e CLT/EBSERH - 36 a 40 horas semanais), dificultando a composição das escalas de trabalho e plantões; (c) as diferenças salariais dos gestores pós EBSERH; $(\mathrm{d})$ diferença do valor pago da insalubridade (pago valor de $5 \%$ a $20 \%$ do vencimento básico para o servidor RJU e para o empregado EBSERH é pago 20 a $40 \%$ do salário base) e ticket de refeição (servidor RJU o valor do Ticket: $\mathrm{R} \$ 1,50$; empregado EBSERH o valor é: R\$4,50), (c). Esse estudo ressalta que, em relação aos cargos de gestão criados pela EBSERH, os gestores possuem uma remuneração média considerada alta, quando comparados aos cargos e funções semelhantes à época em que o Hospital era gerido pela UFES. Os novos gestores possuem uma remuneração superior à anteriormente praticada, sendo importante observar que a EBSERH excluiu os estatutários que antes exerciam o cargo de chefia, o que foi, por vezes, justificado pelo critério da escolaridade, a exemplo da portabilidade do título de Mestre, desconsiderando todo o conhecimento e experiência daqueles que atuavam no HUCAM/UFES já por muitos anos. Essa exclusão parece indicar mais a concepção de que esses funcionários se tornaram obsoletos e incapacitados para prosseguir na função, reforçando o tratamento desigual e a fragmentação dos trabalhadores da instituição.

O estudo D9 destaca que o vínculo CLT dos funcionários EBSERH retirou dos trabalhadores a estabilidade de trabalho, característico do servidor público estatutário, deixando-os vulneráveis aos ditames do capital na esfera da Gestão do Trabalho. O que significa que os contratos EBSERH são considerad os flexíveis e precários quando comparados ao do RJU, pois podem ser rescindidos unilateralmente, a qualquer tempo, inclusive afetando a continuidade da prestação de serviços de saúde. Ademais, a introdução do modelo de gestão EBSERH nos HUF's é direcionada para as práticas de natureza privada, trazendo consigo uma nova cultura organizacional e de produção, em busca do cumprimento de metas, desempenho, resultadose, sobretudo, do lucro.

Portanto, a produção está em primeiro lugar, diminuind o a proteção de emprego com a criação da cultura de exclusão para aqueles que não atenderem aos resultados almejados 
pela EBSERH. Neste sentido, também o estudo D3 concluiu que o modelo EBSERH é voltado para a lógica mercantil, que visa racionalizara organização e a produção do trabalho pormeio da flexibilização da legislação, que estabelece o RJUs como forma de contratação na Administração Pública, com o objetivo de extinguir, progressivamente, os cargos públicos federias RJU dos HUF's.

O estudo D4 reforça esta posição de instabilidade e insegurança nos postos de trabalho, decorrente da coexistência de duplicidade de vínculos, quando diz que a flexibilização e a precarização das relações de trabalho são advindas da aplicação da legislação privada CLT, diversa da estatutária, havendo a possiblidade de demissão do empregado EBSERH, a fim de atender exclusivamente, os objetivos da Empresa.

Além disso, para os estudos D7 e D8, além desvirtuar os serviços públicos com a precarização da forma de contratação de pessoal e serviços, e com a manutenção de distintos regimes num mesmo ambiente profissional, gerando a competitividade funcional, a mudança da natureza jurídica do HUF - de Autarquia para a Empresa Pública - não garantiu os prometidos ganhos de eficiência.

No estudo D10, isto ficou bastante claro nos dizeres do Entrevistado: "Num período mais ou menos médio de tempo, nós não teremos mais no nosso Hospital nenhum servidor RJU, serão todos EBSERH". (FLAUSINO, V.S., 2015, pg. 98). Esta visão reforça a existência de conflitos e contribui para a desintegração da classe trabalhadora e resistência na implementação do modelo de gestão proposto pela EBSERH.

Quanto às relações de poder, os estudos D9, D11 e D13 demonstraram que todos os trabalhadores, independentemente do vínculo contratual, estão subordinados à EBSERH, a qual apresenta uma estrutura hierárquica verticalizada, com a concentração de poder de comando e decisão. Isto dificulta o processo de aceitação e adaptação por parte dos servidores estatutários, por possuírem seus contratos originários com o ente Federal, tornando-se um dos fatores limitadores da gestão.

\section{Condições de trabalho}

Na categoria - Condições de Trabalho - os estudos apontaram os principais fatores que interferiram nos processos e no ambiente de trabalho, bem como aqueles que, direta ou indiretamente, afetaram a vida dos trabalhadores. Demonstraram que as causas como a multiplicidade de vínculos contratuais, direitos distintos, a introdução da gestão gerencial 
focada na produtividade e metas e, sobretudo, a troca de gestordos HUF's, de IFES para EBSERH, impuseram novas condições de trabalho, criando um ambiente dualizado (RJU e EBSERH), competitivo e conflitivo.

No estudo D1, o autor identificou que a implementação do modelo de gestão com foco na produtividade e resultados atingiu de forma negativa os processos e o ambiente de trabalho, ocasionand o conflitos, disputas, competitividade e uma distorção sobre a visão do HUCAM/UFES, de modo que houve a perda da identidade por parte do servidor público estatutário em relação à instituição, tendo sido criado um estereótipo negativo dos servidores, vistos pelos funcionários EBSERH como trabalhadores descompromissados, improdutíveis e resistentes à mudança, o que trouxe consequências negativas, que incluem aumento dos casos de adoecimento desses funcionários.

Já o estudo D10, apontou que o ingresso dos funcionários EBSERH no HU-UFTM também culminou em conflitos, uma vez que eram encarados como invasores territorial, ora eles send o expulsos, ora expulsando profissionais de outras modalidades de setores e turnos. O estudo D11 enfatizou que, no HUOL/UFRN, o ambiente de trabalho tornou-se aflitivo ao ser marcado por conflitos e pouca cooperação entre os pares, chegand o a serpercebid o como violento do ponto de vista psicológico.

Ademais, os estudos D11 e D12 identificaram que a comunicação interna incipiente foi um dos fatores que também contribuíram para a presença deste ambiente hostil de trabalho, assim como a mudança da logomarca UFRN para a EBSERH. Segundo Pessoa (2018), antes da EBSERH, a logomarca era do HUOL/UFRN, um motivo de orgulho para todos os servidores da instituição. Após a EBSERH, havia a presença das duas logomarcas. Mas, atualmente, a logomarca da UFRN tem se tornado cada vez mais rara nos documentos impressos, nos mobiliários e até mesmo nas fachadas do Hospital, retratando um aparente incômodo para os servidores estatutários, causando a ruptura de pertencimento com à instituição.

Finalmente, conforme os estudos $\mathrm{D}_{3}$ e $\mathrm{D}_{4}$, foi construído um novo trabalhador, de caráter individualista e egoísta, ausente do espírito de organização e da integração de classe na luta pelos seus direitos e em busca de melhorias. As relações e condições de trabalho tornaram-se mais frágeis e precárias, ao ponto que os trabalhadores RJU e CLT/EBSERH passaram a se submeter a diversos trabalhos degradantes para si, a fim de se manterem empregados, restando-lhes apenas a opção de acatar as normas de produtividade e o 
cumprimento de metas, pois aqueles que não se enquadrarem ao padrão de excelência definido pela EBSERH serão, possivelmente, excluídos da organização.

\section{Considerações finais}

A criação da EBSERH foi fruto da vontade política, baseada nos princípios da Reforma do Estado iniciada na década de 9o, que buscou, dentre outras coisas, transferiras atividades sociais não exclusivas do Estado para as entidades privadas, bem como reduzir o custo da folha de pagamento de pessoal e o tamanho da máquina pública em nome da eficiência.

Por ser uma empresa pública inserida na Administração Indireta, a EBSERH trouxe consigo a falsa percepção de ser estatal. Contudo, ela é, de natureza, de direito privado que visa à mercantilização dos serviços de saúde dos HUF's, por constituírem a maior rede de saúde de média e alta complexidade do país, atraindo os interesses do capital.

Assim, a implementação da EBSERH nos HUF's é tida como uma privatização ampla ou não clássica, que se revela pela transferência do fundo público em benefício da Empresa EBSERH (GRANEMANN, 2011). Tem por objetivo flexibilizar as normas de direito público e prestigiar a liberdade econômica, sob o argumento errôneo de que o mercado é mais eficiente do que o próprio Estado. Tanto que a saúde e a educação foram deslocadas da ordem de direito social da CF/88, passando a ser reguladas pelos critérios da ordem econômica (CISLAGHI, 2011).

Desta forma, houve a flexibilização dos contratos e direitos trabalhistas. Os novos contratados, empregados da EBSERH, são regidos pelo direito privado, diverso do regime dos servidores públicos, permanecendo a coexistência do hibridismo contratual presente anteriormente à EBSERH. Agora, é possível encontrar, na maioria dos HUF's, servidores estatutários, empregados da EBSERH e os trabalhadores terceirizados via Fundação de Apoio. A ocorrência da multiplicidade dos vínculos trabalhistas (RJU e CLT) e, por consequência, as diferenças legal-trabalhistas como salário, carga horária, valores pagos de insalubridade e ticket de refeição e outros constitui-se, segund o os estudos selecionados, em um fator limitador da gestão, ocasionando conflitos e um ambiente de trabalho hostil. Embora essa diversidade de vínculos já existisse na situação anterior, a presença dos terceirizados via Fundação de Apoio nunca representou ameaça para os servidores públicos, pois sabiam que seus contratos eram por tempo determinado, sendo vistos apenas como 
colaboradores, ao contrário dos empregados EBSERH, cuja presença é vista como ameaça e fonte de frustações.

Ademais, uma parte dos estudos apontou que não houve melhorias na prestação de serviços, uma vez que a EBSERH preza mais pela quantidade do que pela qualidade nos atendimentos. Constatou-se, também, a falta de medicamentos, materiais, roupas de cama e insumos em geral, dificultando a execução das tarefas, bem como um número insuficiente de trabalhadores e leitos para atender ao quantitativo determinado pela Empresa. Isso porque, embora tenha havido novas contratações, a recomposição de pessoal se deu de forma parcial, por vezes, sem planejamento e, na maioria das instituições, ainda não houve a substituição plena dos terceirizados.

Confirmando as previsões dos que criticaram o projeto da EBSERH, o poder de comando e decisão centralizou-se na EBSERH, afastando as IFES das tomadas de decisão com perda da sua autonomia. Ademais, a introdução da gestão gerencial trouxe uma nova cultura organizacional e de produção para a instituição, uma vez que o foco é no resultado e na eficiência, exigindo cada vez mais do trabalhador, o qual é avaliado todo o tempo pelo seu desempenho e cumprimento de metas, de maneira que o ambiente laboral se tornou competitivo e individualizado.

Dito isso, todas essas mudanças criaram condições precárias de trabalho como a presença constante de conflitos, fragmentação e fragilização das categorias profissionais na luta pelos seus direitos e busca de melhorias. Estabelecendo-se a falta de comunicação entre os pares, afastamentos, desligamentos e adoecimentos dos trabalhadores.

Assim, através da Revisão Sistemática de Literatura, foi possível confirmar que o modelo de gestão EBSERH teve efeitos negativos para a gestão dos recursos humanos dos HUF's, frustrando aqueles que acreditavam que as novas contratações e a introdução da nova gestão iriam proporcionar melhorias nos processos e nas condições de trabalho nos HUFs, com reflexos positivos para os seus trabalhadores.

\section{Referências}

ALBUQUERQUE, G. O. Debate sobre a EBSERH em instâncias de controle social: um estudo exploratório. Dissertação (Mestrad em Política Social) - Universidade de Brasília (UnB), 2017 Disponível em: http://repositorio.unb.br/handle/10482/30987 Acesso:5 de dezembro de 2018. 
ANDREAZZI, Maria de Fátima Siliansky. Empresa Brasileira de Serviços Hospitalares: Inconsistências à luz da Reforma do Estado. Revista Brasileira de Educação Médica, p. 275281. Rio de Janeiro, 2013. Disponível em:

http://bases.bireme.br/cgibin/wxislind.exe/iah/online/?IsisScript=iah/iah.xis\&base=LILACS\& lang=p\&nextAction=Ink\&exprSearch=68329g\&indexSearch=ID

AVELAR, J.V.R. Disputa de Frames e Hibridismo Organizacional um conteúdo de caso de um Hospital Universitário Federal. Dissertação (Mestrado em Administração) Universidade Federal do Espírito Santo -UFES. Vitória/E. S, 2016. Disponível em: http://repositorio.ufes.br/handle/10/8895 Acesso: 20 novembro 2018

BARROS, R.T. Empresa Brasileira de Serviços Hospitalares: uma análise sobre sua gestão no contexto institucional do Hospital das Clínicas da UFPE. Dissertação (Mestrado em Gestão Pública) - Universidade Federal de Pernambuco, Recife/PE, 2014. Disponível em: https://repositorio.ufpe.br/handle/123456789/11861 Acesso em: 20 de nov 2018.

BATISTA JR, Francisco. Gestão do SUS: O que fazer? In: BRAVO, Maria Inês Souza [et al]. Saúde na atualidade: porum sistema único de saúde, estatal, Universal, gratuito e de qualidade. Cadernos de Saúde, Rio de Janeiro: UERJ, Rede Sirius/ADURF, 2011. Disponível em: https//www.scrib.com/doc/67170084/revista-cadernos-de-saude Acesso:16/04/2018

BANCO MUNDIAL. Projeto de Reestruturação dos Hospitais Universitários-REHUF. ID; P120391. Folha de dados integrados sobre salvaguardas. Estágio de avaliação. Relatório no AC: 5502 . Site oficial do Banco Mundial. Disponível em: http://www.worldbank.org/en/news/loans-credits/2011/04/05/brazil-federal-universityhospitals-modernization-project Acesso: 16/04/2018.

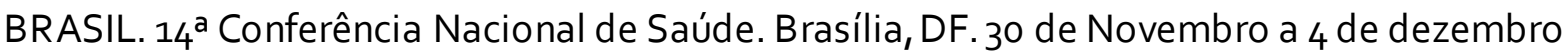

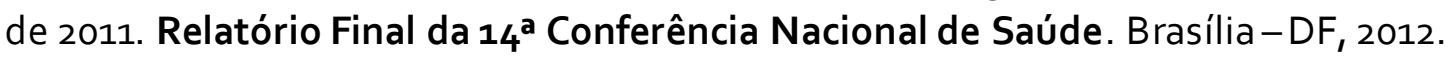
Disponível em: http://bvsms.saude.gov.br/bvs/publicacoes/14_cns_relatorio_final.pdf. Acesso: 15/04/2018.

BRASIL. Decreto-Lei no 7.082, de 27 de janeiro de 2010. Institui o Programa Nacional de Reestruturação dos Hospitais Universitários Federais - REHUF, dispõe sobre o financiamento compartilhado dos Hospitais Universitários Federais entre as áreas da educação e da saúde e disciplina o regime de pactuação global com esses hospitais. Disponível em:

http://www.planalto.gov.br/ccivil 03/ ato20072010/2010/decreto/d7082.htm Acesso: 15/04/2018.

BRASIL, EBSERH.CARTILHA DE PERGUNTASE RESPOSTAS. Disponível em http://www.ebserh.gov.br/web/portalebserh/cartilhas//asset publisher/pgAuovSiacSG/docu ment/id/112496 Acesso: 30 /05/2018.

BRASIL. Lei 12.550 de dezembro de 2011. Autoriza o Poder Executivo a criar a Empresa Pública denominada Empresa Brasileira de Serviços Hospitalares - EBSERH. Disponível em: http://www.planalto.gov.br. Acesso: 15/04/2018. 
BRASIL. Lei Complementar nº10 $^{10}$ de 4 de maio de 2000. Estabelece normas de finanças públicas voltadas para a responsabilidade na gestão fiscal e dá outras providências.

Disponível em: http://www2.camara.leg.br/legin/fed/leicom/200o/leicomplementar-101-4maio-2000-351480-norma-pl.html. Acesso: 21/04/2018.

BRASIL. Tribunal de Contas da União (TCU). Relatório da Auditoria. Entidade: Empresa Brasileira de Serviços Hospitalares -EBSERH.TC032.519/2014-1. Disponível em: http://portal.tcu.gov.br/data/files/5F/14/53/92/52B31510ED8753152A2818A8/032.519\%20E mpresa\%20Servi os\%20Hospitalares\%2ocomp.pdfAcesso: 21/04/2018.

BRESSER-PEREIRA, L.C. Crise Econômica e Reforma do Estado no Brasil: para uma nova interpretação na América Latina. São Paulo: Ed. 34, 1996.

BRAVO, M.I.S. InformAndes. Informativo $n^{0}$ 15, Brasília, 2012. Disponível em: www.andes.org.br/imprensa/noticias/imp-inf-1531188269.pdf Acesso: 21/04/2018.

BRITO, L.M. DE. Satisfação e insatisfação no trabalho de profissionais em Hospital Universitário. Dissertação. Mestrado em Ciências e Tecnologia em Saúde no Programa de Pós-Graduação em Ciências e Tecnologias em Saúde da Universidade de Brasília, 2017. Disponível em: http://repositorio.unb.br/handle/10482/31577 2017 Acesso:10 de dezembro de 2018.

CARDOSO, Renata de Oliveira. O Processo de Contrarreforma do Estado Brasileiro e a Empresa Brasileira de Serviços Hospitalares: a privatização mascarada dos hospitais universitários. In: BRAVO, M.I.S; MENEZES, J.S.B. A Saúde nos Governos do Partido dos Trabalhadores e as Lutas Sociais Contra a Privatização. 1. ed. Rio de Janeiro: UERJ, Rede Sirius, 2014. 98p. Disponível em: http://www.contraprivatizacao.com.br/2014/12/og66.html. Acesso: 15/04/2018.

CARMINATI JR, A. A gestão do Hospital Universitário da Universidade Federal de Santa Maria e da Universidade Federal de Santa Catarina após a adesão à EBSERH.

Dissertação (Mestrado Profissional em Administração - Universidade Federal de Santa Catarina, Santa Catarina, 2017. Disponível em:

https://repositorio.ufsc.br/xmlui/handle/123456789/182796 Acesso: 20 nov de 2018.

CISLAGHI, J.F. Hospitais Universitários Federais e Novos Modelos de Gestão: faces da contrarreforma do Estado no Brasil. In: BRAVO, Maria Inês Souza [et al]. Saúde na atualidade: porum sistema único de saúde, estatal, Universal, gratuito e de qualidade. Cadernos de Saúde, Rio de Janeiro: UERJ, Rede Sirius/ADURF, 2011. Disponível em: https//www.scrib.com/doc/67170084/revista-cadernos-de-saude Acesso: 16/04/2018.

CISLAGHI, J.F. Hospitais Universitários: presente caótico e futuro incerto. Disponível em: https://fopspr.files.wordpress.com/2010/08/hospitais-universitarios.pdf. Acesso: 26/04/2018. 
CORREIA, M.V.C. A Saúde no Contexto da Crise Contemporânea do Capital: o Banco Mundial e as Tendências da Contrarreforma na Política de Saúde Brasileira. Política de Saúde e Serviço Social: Impasses e Desafios Temporalis. Revista da ABEPSS, ano VII, n.13, São Luiz, 2007. Disponível em: http://files.adrianonascimento.webnode.com.br. Acesso: $16 / 04 / 2018$.

CORREIA, M.V.C. Porque ser contra aos novos modelos de gestão do SUS? In: BRAVO, Maria Inês Souza [et al]. Saúde na atualidade: porum sistema único de saúde, estatal, Universal, gratuito e de qualidade. Cadernos de Saúde, Rio de Janeiro: UERJ, Rede Sirius/ ADURF, 2011. Disponível em: https//www.scrib.com/doc/67170084/revista-cadernos-desaude Acesso: 16/04/2018.

DI PIETRO.M.S.Z. Parcerias na Administração Pública. 11. ed. Rio de Janeiro: Forense, 2017.

DRUCK, Graça. A Terceirização na saúde pública: formas diversas de precarização do trabalho.Trab. educ. saúde[online]. 2016, vol.14, suppl.1, pp.15-43. ISSN 16781007. http://dx.doi.org/10.1590/1981-7746-soloo023. Disponível em: http://www.scielo.br/scielo.php?pid=S198177462016000400015\&script=sci abstract\&tlng= pt Acesso: 21/04/2018.

FARIA, P.M. Revisão Sistemática da Literatura: contributo para um novo paradigma investigativo. Metodologia e procedimentos na das ciências e educação. 1. ed. Santo Terso, fev de 2016.

FERNANDES, R.B. Da Conquista ao Desmonte: um estudo sobre os descaminhos da política de saúde em tempos de EBSERH. Dissertação (Mestrado em Política Pública) Universidade de Brasília, Brasília/DF, 2017. Disponível em: http://repositorio.unb.br/handle/10482/23732 Acesso: 20 de nov de 2018.

FLAUSINO, V.S. Cultura e poder na organização hospitalar: as relações de poderna implantação da EBSERH e um Hospital Universitário. Dissertação (Mestrado em Ciências Sociais) - Universidade Federal de Uberlândia - UFTM, Uberlandia/MG, 2015.Disponível em: https://repositorio.ufu.br/handle/123456789/12011 Acesso em: 20 de nov 2018.

GRANEMANN, S. Fundações Estatais: Projeto de Estado do Capital. In: BRAVO, Maria Inês Souza [et al]. Saúde na atualidade: por um sistema único de saúde, estatal, Universal, gratuito e de qualidade. Cadernos de Saúde, Rio de Janeiro: UERJ, Rede Sirius/ ADURF, 2011. Disponível em: https//www.scrib.com/doc/67170084/revista-cadernos-de-saude Acesso: 16/04/2018.

GRAGNOLATI, M.; LINDELOW, M.; COUTTOLENC, B. 20 Anos de Construção do Sistema Único de Saúde no Brasil: uma análise do Sistema Único de Saúde. The World Bank, Washington, D.C., 2013. Disponível em: < https://openknowledge.worldbank.org/bitstream/handle/10g86/15801/PORT TYHSRB WEB FULL.pdf? sequence $=5$. Acesso em: 23 de maio de 2018. 
GURGEL, J.L.M. Análise de desempenho: um estudo aplicado em um Hospital Universitário do Rio Grande do Norte conveniado à EBSERH. Dissertação (Mestrado em Engenharia da Produção) - Universidade Federal do Rio Grande do Norte - UFRN. Natal, RN, 2016. Disponível em: https://repositorio.ufrn.br/jspui/handle/12345678g/23785 Acesso: 20 de nov 2018.

LITTIKE, Denilda; SODRÉ, Francis. A Arte Do Improviso: o processo de trabalho dos gestores de um Hospital Universitário Federal. Ciênc. saúde coletiva [online]. 2015, vol.20, n.10, pp.3051corr3062. ISSN 1413-8123. Disponível

em: http://www.scielo.br/scielo.php?pid=S141381232015001003051\&script=sci_abstract\&tl ng=pt. Acesso em: 26/04/2018.

MARINHO, R.N. Implicações estruturais e comportamentais na implementação do modelo de gestão da EBSERH em um Hospital Universitário. Dissertação (Mestrado em Administração)-Universidade Federal do Espírito Santo, Vitória/ES, 2016. Disponível em: http://repositorio.ufes.br/handle/10/8893 Acesso: 20 de nov 2018.

MAZETO, Y.E.S. Política de saúde face aos avanços neoliberais e a implantação da Empresa Brasileira de Serviços Hospitalares. Dissertação (Mestrado em Serviço Social PUC-GOIAS, 2017. Disponível em: https://repositorio.ufjf.br/jspui/handle/ufjf/3668 Acesso 20 de nov 2018.

OLIVEIRA, G. DE A. A compatibilidade dos princípios e modelo de estado que subjazem ao SUS e a EBSERH. Dissertação (Mestrado em Ciências Sociais) - Faculdade de Filosofia da Pontifícia Universidade Católica do Rio Grande do Sul, 2014. Disponível em: http://hdl.handle.net/10923/5849> Acesso: 10 de dezembro de 2018.

ORLANDI, Daniele de Paula. Análise dos impactos da EBSERH no desempenho dos Hospitais Universitários Federais. Dissertação. (Mestrado Profissional em Gestão Pública) - Universidade Federal do Espírito Santo, 2016. Disponível em:http://repositorio.ufes.br/handle/10/8676 Acesso: 10 de dezembro de 2018.

PAULA, R.B. DE. Desafios de Gestão do HU/UFSC, após a criação da EBSERH pelo Governo Federal. (Mestrado Profissional em ) - Universidade Federal de Santa Catarina, 2016. Disponível em:

https://repositorio.ufsc.br/xmlui/bitstream/handle/12345678g/175078/344483.pdf?sequence =1\&isAllowed=y Acesso: 10 de dezembro de 2018.

PESSOA, C.M. Cultura Organizacional e Condições de Trabalho: impactos da contratualização de um Hospital Universitário com a EBSERH. Dissertação (Mestrado em Gestão Pública) - Universidade Federal do Rio Grande do Norte, Natal, RN, 2018. Disponível em: https://repositorio.ufrn.br/jspui/handle/12345678g/25556

PINHEIRO, K.F. Os Efeitos da EBSERH na Gestão dos Recursos Humanos dos Hospitais Universitários Federais. Dissertação (Mestrado em Políticas Sociais e Cidadania) Universidade Católica de Salvador(UCSAL), Salvador, 2019. 
SANT'HELENA, M.M.; PASSOS, A.F.; COSTA, C.B.; KRUGER, T.R. A Reforma Sanitária às Privatizações: discutindo a saúde pública brasileira. Congresso Catarinense de Assistentes Sociais. Agosto, 2013.Florianóplolis/SC. Disponível em: file http://cress-sc.org.br/wpcontent/uploads/2014/03/Da-Reforma-Sanit\%C3\%A1ria-\% $\% \mathrm{C}_{3} \%$ AosPrivatiza\% $\mathrm{C}_{3} \% \mathrm{~A} 7 \% \mathrm{C}_{3} \% \mathrm{~B}_{5}$ es-discutindo-a-sa\%C3\%BAde-p\%C3\%BAblica-brasileira.pdf. Acesso: 21/04/2018.

SANTOS, Agnaldo Castilho; OLIVEIRA JR, Lourival Batista. REHUF: uma ferramenta para tomada de decisão e sua aplicação na saúde coletiva. HU Revista, Juiz de Fora, v. 42, n. 1, p. 43-51, jan./jun. 2016. Disponível em:

https://hurevista.ufjf.emnuvens.com.br/hurevista/article/view/2397 Acesso: 16/04/2015.

SILVA, J.A. Empresa Brasileira de Serviços Hospitalares: coerência ou contradição de um Estado empresário? Dissertação (Mestrado do Programa em Administração e Ciências Contábeis) - Universidade Federal Fluminense Nitéroi/RJ, 2016. Disponível em: https://app.uff.br/riuff/handle/1/2164 Acesso: 20 nov de 2018.

SODRÉ, F.; LITTIKE, D.; DRAGO, L.M.B.; PERIM, M.C.M. Empresa Brasileira de Serviços Hospitalares: um novo modelo de gestão?. Serv. Soc. Soc., São Paulo, n.114, p.365-380, abr/jun, 2013. Disponível em:

http://www.scielo.br/scielo.php?script=sci arttext\&pid=S0101-66282013000200009 Acesso: 21/04/2018.

TEIXEIRA, Enio Henrique. Um sistema de qualidade para a gestão compartilhada do Hospital Universitário da UFJF com a EBSERH. Dissertação (Mestrado Profissional em Gestão e Avaliação da Educação) - Universidade Federal de Juiz de Fora (CAEd/UFJF). Juiz de Fora/M. G, 2016. Disponível em: https://repositorio.ufjf.br/jspui/handle/ufjf/3668 Acesso: 20 nov 2018.

VIEIRA, K.R. Transformações estruturais e institucionais da gestão do HULW/UFPB. Dissertação (Mestrado em Administração) - Universidade Federal do Rio Grande do Norte UFRN) Natal /RN, 2017. Disponível em:

https://repositorio.ufrn.br/jspui/handle/12345678g/24216 Acesso: 20 nov 2018.

ZIGATO, J.O. A Implantação da Empresa Brasileira de Serviços Hospitalares (EBSERH) no Hospital Universitário da Universidade Federal de Juiz de Fora (HU-UFJF): dilemas presentes e perspectivas futuras. Dissertação (Mestrado). Orientadora: Professora Doutora Lêda Maria Leal de Oliveira - Universidade Federal de Juiz de Fora - MG. Pós-Graduação em Serviço Social., 2015. Disponível em: https://repositorio.ufjf.br/jspui/handle/ufjf/1128 Acesso em: 20/05/2018. 


\section{Dados das autoras}

Karina de Farias Pinheiro

Mestre em Políticas Sociais e Cidadania pela Universidade Católica de Salvador - UCSAL. E-mail: : karinapinheiro@gmail.com

\section{Ângela Maria Carvalho Borges}

Doutora em Ciências Sociais pela UFBA, docente do PPG Políticas Sociais e Cidadania, UCSAL. E-mail: angela.borges@pro.ucsal.br 